\title{
Factores pronósticos y sobrevida en pacientes menores de 18 años con tumores de la familia del sarcoma de Ewing: experiencia de 10 años
}

\author{
Ligia Rios $^{1, a}$, Liliana Vásquez ${ }^{1, a}$, José María Silva ${ }^{2, a}$, Luis Sialer ${ }^{2, a}$, Iván Maza ${ }^{1, a}$, Mónica Oscanoa $^{1, a}$, Gloria Paredes ${ }^{1, a}$, Jenny Gerónimo ${ }^{1, a}$ \\ RESUMEN
}

Objetivo: Describir las características clínicas y epidemiológicas, y determinar los factores pronósticos, sobrevida libre de evento (SLE) y sobrevida global (SG) de los pacientes con tumores de la familia del Sarcoma de Ewing (TFSE).

Materiales y métodos: Estudio retrospectivo llevado a cabo en pacientes menores de 18 años con TFSE, atendidos en la Unidad de Oncología Pediátrica y del Adolescente del Hospital Edgardo Rebagliati entre 2006 y 2016. El análisis descriptivo se realizó mediante distribución de frecuencias. Para el análisis de SLE y SG se utilizaron las curvas de Kaplan-Meier. Se efectuó un análisis univariado y multivariado según modelo de regresión de Cox para variables demográficas, clínicas y quirúrgicas, y factores pronósticos. La medida de fuerza de asociación se expresó en hazard ratio (HR) e intervalo de confianza al 95\% (IC $95 \%)$, y se consideró $\mathrm{p}<0.05$ para diferencias significativas.

Resultados: Se presentaron 29 casos de TFSE. La mediana de edad fue de 9 años (rango 2-17), el 55\% fueron varones. La localización más frecuente fue la pelvis (31\%). El 59\% presentaron metástasis al diagnóstico. La SLE a 3 años en TFSE localizados fue del $40.4 \%( \pm 14.4 \mathrm{EE})$ y con metástasis, $14.6 \%( \pm 12.2$, EE). La SG a 3 años en TFSE localizados fue del $53.9 \%( \pm 17.8 \mathrm{EE})$ y en enfermedad metastásica, $15.1 \%( \pm 9.7, E E)$. El tamaño tumoral $\geq 5 \mathrm{~cm}(\mathrm{HR} 14.84, \mathrm{p}=0.01)$ y la presencia de metástasis al debut (HR 3.23, $p=0.01$ ) fueron factores pronósticos independientes de peor SG. No hubo diferencia significativa en relación con el pronóstico según el sexo, edad, tipo histológico, compromiso de los bordes quirúrgicos o localización del tumor.

Conclusiones: Los TFSE son altamente agresivos. Los factores pronósticos que contribuyen a una menor SLE y SG son la presencia de metástasis al debut de la enfermedad y un tamaño tumoral $\geq 5 \mathrm{~cm}$.

Palabras clave: Sarcoma de Ewing; tumores neuroectodérmicos periféricos primitivos; pronóstico; sobrevida (Fuente: DeCS BIREME).

\section{Prognostic factors and survival in patients under 18 years of age with Ewing sarcoma family tumors: a 10-year experience}

\section{ABSTRACT}

Objective: To describe the clinical and epidemiological characteristics, and to determine prognostic factors, event-free survival (EFS) and overall survival (OS) of patients with Ewing sarcoma family tumors (ESFT).

Materials and methods: A retrospective study conducted in patients under 18 years of age with ESFT, treated at the Unit of Pediatric and Adolescent Oncology of the Hospital Edgardo Rebagliati between January 2006 and June 2016. The descriptive analysis was performed by frequency distribution. Kaplan-Meier curves were used for EFS and OS analysis. Univariate and multivariate analyses were performed according to the Cox regression model for demographic, clinical and surgical variables, and prognostic factors. The measure of strength of association was expressed in hazard ratio (HR) and $95 \%$ confidence interval $(95 \% \mathrm{Cl})$, and $\mathrm{p}<0.05$ was considered for significant differences.

Results: There were 29 cases of ESFT. The median age was 9 years (range 2-17), 55\% were males. The most frequent location was the pelvis (31\%). Fifty-nine percent (59\%) had metastasis at diagnosis. The 3-year EFS in localized ESTF was $40.4 \%( \pm 14.4 \mathrm{EE})$ and with metastasis was $14.6 \%( \pm 12.2$, EE). The 3 -year OS in localized ESTF was $53.9 \%$ ( $\pm 17.8 \mathrm{EE})$ and with metastatic disease was $15.1 \%( \pm 9.7, E E)$. The tumor size $\geq 5 \mathrm{~cm}(\mathrm{HR} 14.84, \mathrm{p}=0.01)$ and the presence of metastasis at the onset of the disease (HR 3.23, $p=0.01)$ were independent prognostic factors of worse OS. There was no significant difference in prognosis regarding the gender, age, histological type, involvement of surgical borders or location of the tumor.

Conclusions: ESFT are highly aggressive. The prognostic factors that contribute to a lower EFS and OS are the presence of metastasis at the onset of the disease and a tumor size $\geq 5 \mathrm{~cm}$.

Keywords: Ewing sarcoma; neuroectodermal tumors; primitive; peripheral; prognosis; survivorship (Source: MeSH NLM).

1. Oncología Pediátrica y del Adolescente.

2. Ortopedia Oncológica.

a. Hospital Nacional Edgardo Rebagliati Martins. Lima, Perú. 


\section{INTRODUCCIÓN}

Los tumores de la familia del Sarcoma de Ewing (TFSE) definen un grupo de neoplasias agresivas de origen neuroectodérmico, que incluyen el Sarcoma de Ewing (SE), Tumor de Askin de la parrilla costal y el tumor neuroectodérmico primitivo (PNET) ${ }^{(1)}$. Histológicamente los TFSE comprenden neoplasias de células pequeñas redondas, que en su mayor parte expresan CD99, y que se caracterizan por presentar una traslocación cromosomal específica que implica el gen EWSR1 en el cromosoma 22, con un gen de transformación del virus de la eritroblastosis (FLI1 en el $85 \%$ de los casos) ${ }^{(1)}$. En el gen de Fusión EWS/ FLI1 $[\mathrm{t}(11 ; 22)(\mathrm{q} 24 ; \mathrm{q} 12)]$, está presente en $>95 \%$ de los sarcomas de la familia de Ewing ${ }^{(2)}$.

El Sarcoma de Ewing es el segundo tumor óseo primario más común en niños y adolescentes por detrás del osteosarcoma, representa alrededor del $1.5 \%$ de todas las neoplasias en los niños menores de 15 años, la incidencia actual es de aproximadamente 2.9 por millón de habitantes menores de 20 años, tiene un pico en la 2da década de la vida, siendo infrecuente en menores de 5 años y en mayores de 30 años, el SE es un poco más prevalente en varones, con un ratio de $1,3 / 1$ y en pacientes de raza blanca ${ }^{(2)}$.

A nivel nacional los TFSE son tumores de baja incidencia, según el Registro de Cáncer de Lima Metropolitana ${ }^{(3)}$, la prevalencia de tumores óseos (incluidos Osteosarcomas y Sarcomas de Ewing) en los pacientes entre los 0 y 14 años era del $3.9 \%$ en los varones y del $3.5 \%$ en mujeres, ocupando el 5to y 8vo lugar respectivamente; por su parte en el grupo conformado por los adolescentes y adultos jóvenes (15 a 29 años), fue del 4.5\% para los varones.

Múltiples estudios han identificado factores pronósticos clínicos en TFSE. La presencia de metástasis en el momento del diagnóstico es consistentemente el factor pronóstico clínico adverso más fuerte ${ }^{(4-6)}$.

Un mayor tamaño del tumor y una mayor edad también se asocian consistentemente con un mal pronóstico ${ }^{(4)}$. También se han evaluado otros factores pronósticos, incluyendo el sitio del tumor, el origen del tejido (hueso o tejido blando) y la raza/etnia. Sin embargo, no se ha logrado un consenso claro sobre su valor pronóstico independiente ${ }^{(7)}$.

Aunque estos estudios han identificado factores que individualmente impactan el pronóstico de los TFSE, han sido desarrolladas en países desarrollados, siendo pocas publicaciones los han descritos en países hispanos ${ }^{(8)}$. Debido a la poca información en nuestro medio referente a esta patología, se decide realizar este estudio con el fin de describir las características clínicas, epidemiológicas, y determinar los factores pronósticos, sobrevida libre de enfermedad y sobrevida global de los pacientes con tumores de la familia del Sarcoma de Ewing atendidos en la Unidad de Oncología Pediátrica del Hospital Nacional Edgardo Rebagliati Martins entre los años 2006 y 2016.

\section{MATERIALES Y MÉTODOS}

Se realizó un estudio de tipo cohorte retrospectiva, donde se analizaron los datos obtenido de las historias clínicas del Hospital Nacional Edgardo Rebagliati Martins (HNERM), en Lima-Perú. La población estuvo conformada por todos los pacientes menores de 18 años, que fueron diagnosticados con TFSE, entre enero del 2006 y junio del 2016 en la Unidad de Oncología Pediátrica y del Adolescente en el HNERM.

El análisis descriptivo se realizó mediante una tabla de distribución de frecuencias. Para el análisis de sobrevida libre de evento (SLE) se consideró como el tiempo desde el momento del diagnóstico hasta la fecha de progresión, recurrencia, muerte o último contacto, mientras que la sobrevida global (SG), se consideró el tiempo transcurrido desde el diagnóstico hasta la muerte o último contacto, para esto se utilizaron las curvas de Kaplan-Meier y Logrank test.

Se analizaron los datos de variables demográficas, clínicas, quirúrgicas y otros factores pronósticos conocidos mediante análisis univariado y multivariado según modelo de regresión de Cox. La medida de fuerza de asociación se expresó en hazard ratio (HR), con un intervalo de confianza al 95\% (IC 95\%). Se consideró un nivel de $\mathrm{p}<0.05$ para diferencias significativas. Se utilizó el paquete estadístico STATA statistical package (Small STATA Version 13.0, Stata Corporation, College Station, Texas, USA).

\section{RESULTADOS}

Entre enero del 2006 y junio del 2016 se registraron 29 casos de niños y adolescentes con diagnóstico confirmado de TFSE. La mediana de edad fue de 9 años (rango 2-17 años) y el $79.3 \%$ de los pacientes tenía menos de 15 años. La mayoría de pacientes fueron del sexo masculino (55\%) y procedían de la ciudad de Lima. La localización primaria más común fue la pelvis (31\%). Dieciséis pacientes (55\%) desarrollaron enfermedad ósea, los restantes se presentaron en ubicaciones diversas como renal, cervical, abdominal y tejido celular subcutáneo. El PNET o tumor neuroectodérmico primitivo fue el tipo histológico más frecuente, seguido del sarcoma de Ewing y Tumor de Askin (52\%, 41\% y 7\% respectivamente). El 59\% de los pacientes presentó enfermedad metastásica al debut. El $100 \%$ de los pacientes recibió tratamiento quimioterápico neoadyuvante, el $65 \%$ recibió tratamiento local combinado con radioterapia y cirugía, y el $13 \%$ no recibió ningún tratamiento local adyuvante. El $58 \%$ de los pacientes presentó un estadio clínico IV al debut de la enfermedad (Tabla 1). 
Factores pronósticos y sobrevida en pacientes menores de 18 años con tumores de la familia del sarcoma de Ewing: experiencia de 10 años

Tabla 1. Características basales de la población estudiada

\begin{tabular}{|c|c|c|c|}
\hline \multirow[t]{2}{*}{ Característica } & Total $(\mathrm{N}=29)$ & \multirow[b]{2}{*}{$\mathrm{N}$} & \multirow[b]{2}{*}{$\%$} \\
\hline & & & \\
\hline \multicolumn{4}{|c|}{ Edad } \\
\hline & Mediana años (rango) & $9.4(2-17)$ & \\
\hline & $<15$ años & 23 & 79.3 \\
\hline & $\geq 15$ años & 6 & 20.7 \\
\hline \multicolumn{4}{|c|}{ Sexo } \\
\hline & Masculino & 16 & 55.17 \\
\hline & Femenino & 13 & 44.83 \\
\hline \multicolumn{4}{|c|}{ Lugar Primario } \\
\hline & Pelvis & 9 & 31.0 \\
\hline & Extremidad inferior & 6 & 20.7 \\
\hline & Tórax & 4 & 13.8 \\
\hline & Tejidos blandos y otros & 4 & 13.8 \\
\hline & Cabeza y cuello & 3 & 10.3 \\
\hline & Columna & 2 & 6.9 \\
\hline & Extremidad superior & 1 & 3.4 \\
\hline \multicolumn{4}{|c|}{ Lugar Primario } \\
\hline & Pélvico & 9 & 31.03 \\
\hline & No pélvico & 20 & 68.97 \\
\hline \multicolumn{4}{|c|}{ Histología } \\
\hline & PNET & 15 & 51.74 \\
\hline & SE & 12 & 41.38 \\
\hline & Askin & 2 & 6.88 \\
\hline \multicolumn{4}{|c|}{ Óseo/extraóseo } \\
\hline & Óseo & 16 & 55.17 \\
\hline & Extraóseo & 13 & 44.83 \\
\hline \multicolumn{4}{|c|}{ Enf. Metastásica } \\
\hline & Sí & 17 & 58.62 \\
\hline & No & 12 & 41.38 \\
\hline \multicolumn{4}{|c|}{ Tamaño tumoral } \\
\hline & $>=5 \mathrm{~cm}$ & 21 & 72.41 \\
\hline & $<5 \mathrm{~cm}$ & 8 & 27.41 \\
\hline \multicolumn{4}{|c|}{ Bordes quirúrgicos } \\
\hline & Libres & 16 & 55.17 \\
\hline & Comprometidos & 8 & 27.59 \\
\hline & No aplica & 5 & 17.24 \\
\hline \multicolumn{4}{|c|}{ Tratamiento local } \\
\hline & Radioterapia sola & 3 & 10.34 \\
\hline & Radioterapia y cirugía & 19 & 65.52 \\
\hline & Cirugía sola & 3 & 10.34 \\
\hline & No tratamiento local & 4 & 13.80 \\
\hline \multicolumn{4}{|c|}{ Estadio clínico } \\
\hline & Estadio II & 3 & 10.34 \\
\hline & Estadio III & 9 & 31.03 \\
\hline & Estadio IV & 17 & 58.62 \\
\hline
\end{tabular}


En el análisis del Log-rank test, la ubicación pélvica del tumor y tamaño del tumor $\geq 5 \mathrm{~cm}$ fueron estadísticamente significativos para menor SG y sólo el tamaño tumoral $\geq 5 \mathrm{~cm}$ fue estadísticamente significativo para menor SLE (Tabla 2).

Tabla 2. Log-rank test para SG y SLE

$\begin{array}{rcc} & \begin{array}{c}\text { Log Rank }(p) \\ \text { SG }\end{array} & \begin{array}{c}\text { Log Rank }(p) \\ \text { SLE }\end{array} \\ \text { Metástasis } & 0.08 & 0.70 \\ \text { Pélvico o no } & 0.03 & 0.28 \\ \text { Tamaño >=5cm } & 0.01 & 0.01 \\ \text { Bordes qx } & 0.65 & 0.47 \\ \text { Tratamiento local } & 0.75 & 0.08 \\ \text { Axial vs extremidad } & 0.79 & 0.44 \\ \text { Sexo } & 0.94 & 0.57 \\ \text { Edad>=15 años } & 0.50 & 0.30 \\ \text { Histología } & 0.41 & 0.68 \\ \text { Estadio clínico } & 0.16 & 0.07 \\ \text { Óseo vs extra óseo } & 0.08 & 0.91\end{array}$

La SLE a 3 años en los pacientes con TFSE localizado, es decir sin metástasis, fue del $40.4 \%$ ( \pm 14.4 Estándar Error, EE), y en enfermedad metastásica del 14.6\% ( \pm 12.2 , EE). La mediana de seguimiento fue 37.5 meses (rango 2-123). La SG a 3 años en los pacientes con TFSE localizado fue del 53.9\% ( $\pm 17.8 \mathrm{EE})$, y en enfermedad metastásica del $15.1 \%( \pm 9.7, \mathrm{EE})$ (Figura 1).



Figura 1. Sobrevida global en años de acuerdo a la presencia de Metástasis

El tamaño tumoral $\geq 5 \mathrm{~cm}$ (Hazard Ratio, HR 5.39, $\mathrm{p}=0.02$ ) fue un factor significativo para una peor SLE, tanto en el análisis univariado como multivariado (Tabla 3 y Figura 2). 
Factores pronósticos y sobrevida en pacientes menores de 18 años con tumores de la familia del sarcoma de Ewing: experiencia de 10 años

Tabla 3. Análisis de riesgo proporcional por Regresión de Cox para sobrevida libre de enfermedad en pacientes con tumores de la familia de Sarcoma de Ewing

\begin{tabular}{|c|c|c|c|c|}
\hline \multirow[t]{2}{*}{ Variable } & Univariado & & Multivariado & \\
\hline & $\begin{array}{l}\text { Hazard Ratio } \\
\text { (IC 95\%) }\end{array}$ & p-valor & $\begin{array}{l}\text { Hazard Ratio } \\
\text { (IC } 95 \% \text { ) }\end{array}$ & p-valor \\
\hline Sexo masculino & $0.76(0.29-1.99)$ & 0.58 & & \\
\hline Edad >=15 años & $0.48(0.11-2.11)$ & 0.33 & & \\
\hline Metástasis inicial & $1.19(0.46-3.06)$ & 0.70 & $1.40(0.51-3.82)$ & 0.56 \\
\hline Pélvico vs no pélvico & $1.72(0.61-4.82)$ & 0.30 & & \\
\hline Axial vs extremidad & $0.69(0.26-1.80)$ & 0.46 & & \\
\hline Tamaño tumoral $\geq 5 \mathrm{~cm}$ & $5.09(1.14-22.6)$ & 0.01 & $5.39(1.19-24.46)$ & 0.02 \\
\hline $\begin{array}{r}\text { Bordes quirúrgicos } \\
\text { comprometidos }\end{array}$ & $1.45(0.50-4.22)$ & 0.49 & & \\
\hline Óseo vs extra óseo & $1.05(0.41-2.66)$ & 0.91 & & \\
\hline Estadio clínico & $1.55(0.81-2.96)$ & 0.17 & $1.61(0.78-3.31)$ & 0.19 \\
\hline
\end{tabular}

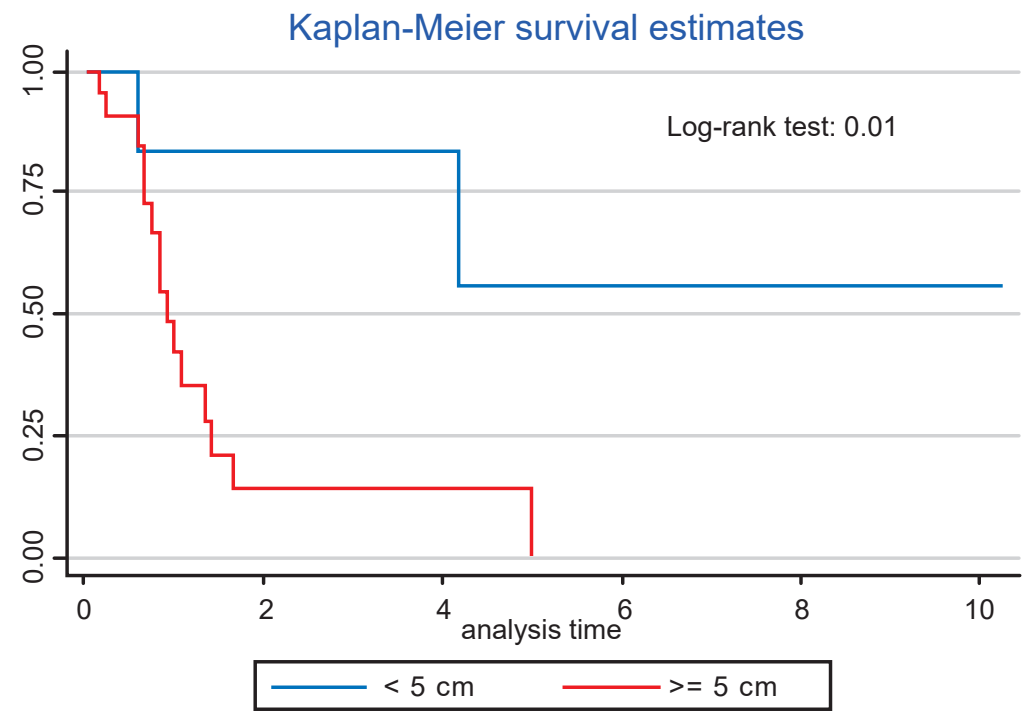

Figura 2. Sobrevida libre de enfermedad en años de acuerdo al tamaño tumoral

Se pudo demostrar en el análisis univariado y multivariado, que el tamaño tumoral $\geq 5 \mathrm{~cm}$ (Hazard Ratio, HR 14.84, $\mathrm{p}=0.01$ ) y la presencia de metástasis al debut (HR 3.23, p=0.01) fueron factores pronósticos independiente de peor SG (Tabla 4 y Figura 3 ). 
Tabla 4. Análisis de riesgo proporcional por Regresión de Cox para sobrevida global en pacientes con tumores de la familia de Sarcoma de Ewing

\begin{tabular}{rccc}
\multicolumn{1}{c}{ Variable } & $\begin{array}{c}\text { Univariado } \\
\text { Hazard Ratio } \\
(\text { IC } 95 \%)\end{array}$ & p-valor & Multivariado \\
Sexo masculino & $0.96(0.38-2.45)$ & 0.94 & $\begin{array}{c}\text { Hazard Ratio } \\
\text { (IC 95\%) }\end{array}$ \\
Edad >= 15 años & $0.61(0.54-3.65)$ & 0.51 & \\
Metástasis inicial & $2.26(0.84-6.05)$ & 0.09 & $3.23(1.05-9.92)$ \\
Pélvico vs no pélvico & $2.71(1.03-7.15)$ & 0.04 & $1.10(0.74-5.43)$ \\
Tamaño tumoral>5cm & $10.36(1.35-79.2)$ & 0.01 & $14.84(1.83-120)$ \\
Bordes quirúrgicos & $1.25(0.44-3.55)$ & 0.66 & 0.01 \\
comprometidos & $0.43(0.16-1.17)$ & 0.09 & \\
Óseo vs extra óseo & $2.00(0.93-4.31)$ & 0.07 & $1.81(0.61-5.33)$ \\
Estadio clínico & & & 0.27
\end{tabular}

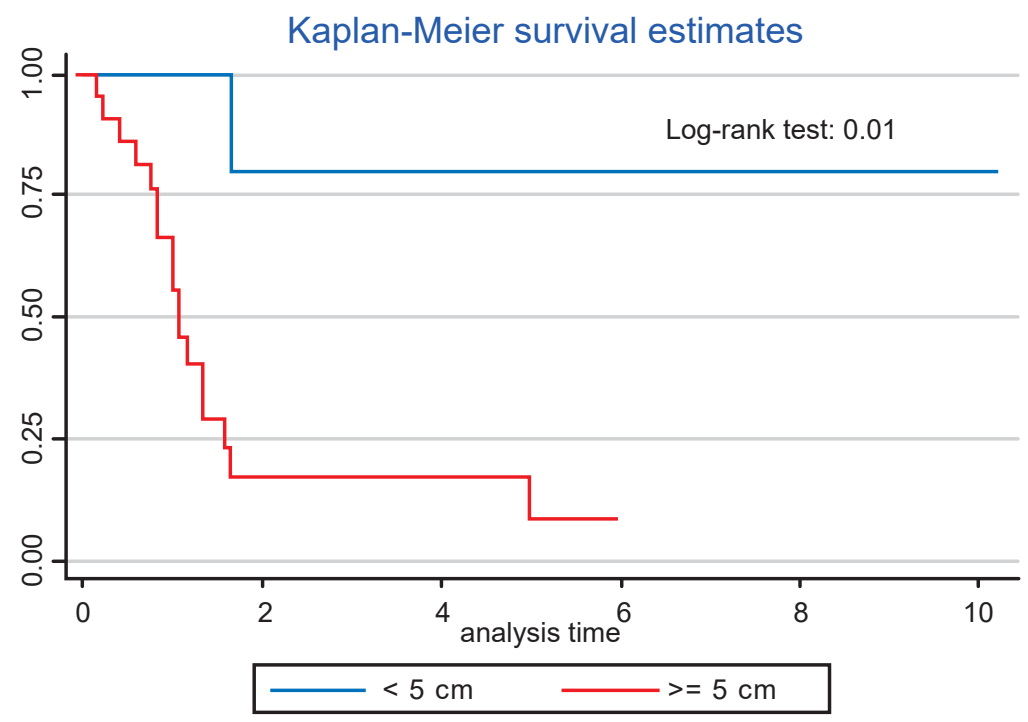

Figura 3. Sobrevida global en años de acuerdo al tamaño tumoral

En el análisis univariado la ubicación pélvica (HR 2.71, $\mathrm{p}=0.04)$ fue un factor pronóstico para menor SG, sin embargo esto no se mantuvo en el análisis multivariado (Figura 4). Tampoco hubo diferencia significativa en relación a pronóstico según el sexo, edad, tipo histológico, compromiso de los bordes quirúrgicos, localización del tumor, o si era óseo o extraóseo. 


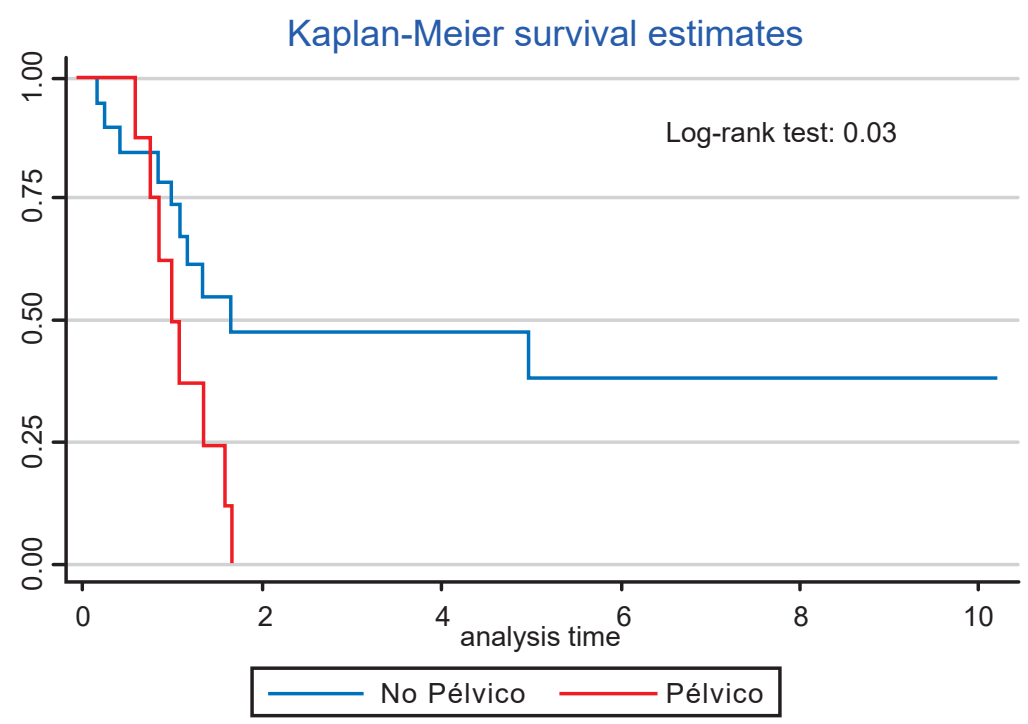

Figura 4. Sobrevida global en años de acuerdo a la ubicación (pélvica o no pélvica)

Por otra parte en lo referente al estadio clínico, este no fue estadísticamente significativo para la SLE (univariado $\mathrm{p}=0.17$ y multivariado $p=0.19$ ) ni tampoco para la SG (univariado $p=0.07$ y multivariado $p=0.27$ ). La SG de los pacientes con estadio clínico IV fue 0.13 (Figura 5).

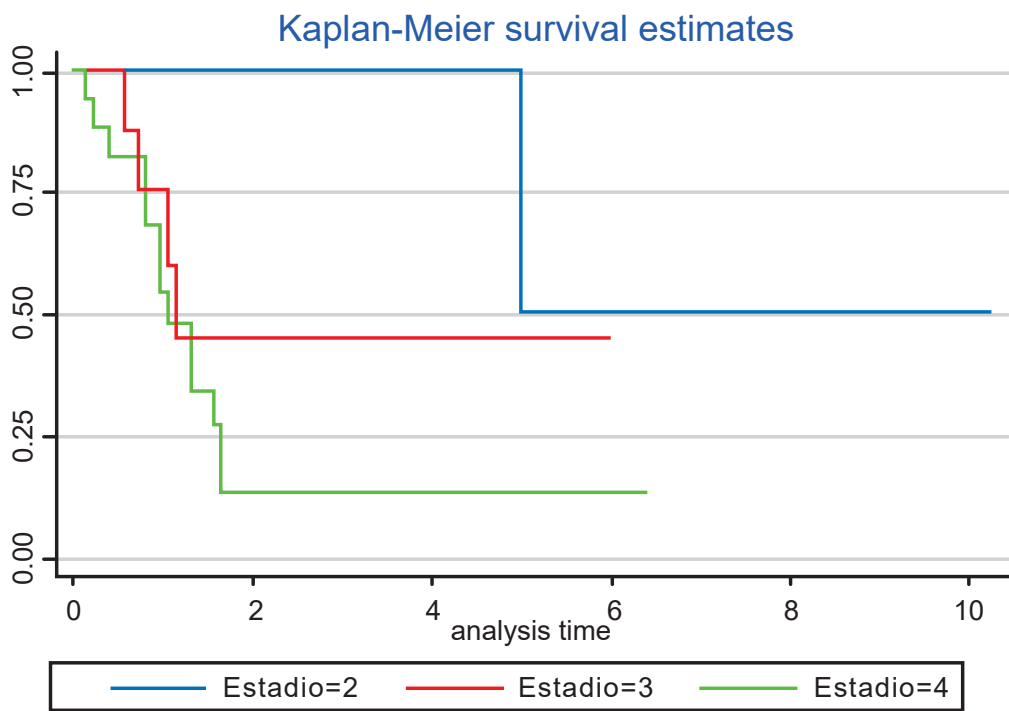

Figura 5. Sobrevida global en años de acuerdo al estadio clínico

\section{DISCUSIÓN}

Los TFSE son neoplasias que se manifiestan casi exclusivamente en la población pediátrica y adolescente, suelen desarrollarse por lo general en el tejido óseo como Sarcoma de Ewing, pero también se han presentado casos a nivel extraóseo ${ }^{(6,9)}$, cutáneo ${ }^{(10)}$, renal ${ }^{(11)}$ entre otras ubicaciones. A pesar de los avances en el tratamiento quimioterápico como en el tratamiento local de esta enfermedad, no se ha logrado aumentar la sobrevida sustancialmente en los últimos años y se ha observado en varios estudios que existen factores pronósticos adversos que disminuyen aún más esta sobrevida.

Dentro de los resultados obtenidos en la presente investigación, encontramos que los TFSE fueron más 
frecuentes en los varones (55\%), similar a lo reportado en la literatura internacional ${ }^{(4,6,12,13)}$, y la ubicación más frecuente fue la pelvis, tal como lo describió Cotterill et al en el European Intergroup Cooperative Ewing's Sarcoma Study Group $^{(5)}$. Vemos que casi el $80 \%$ de los pacientes evaluados tenía menos de 15 años en el momento del diagnóstico. Pudimos observar que ni el sexo ni la edad mayor o igual a 15 años, demostraron ser factores pronósticos, a diferencia de lo publicado por el Instituto Rizzolli ${ }^{(14)}$, donde en su análisis univariado el ser varón y tener más de 14 años fueron considerados como factores de mal pronóstico, sin embargo en su análisis multivariado sólo el sexo masculino se mantuvo como factor independiente de mal pronóstico. En nuestro estudio la presencia de metástasis al debut de la enfermedad se evidenció en el $58.6 \%$ de los pacientes, cifra muy superior a los estudios de Karski et al ${ }^{(7)}$ con $28 \%$, y Duchman et al ${ }^{(15)}$ con $32 \%$, realizados en población estadounidense y a lo publicado por Raciborska et al ${ }^{(13)}$ que encontró $37.8 \%$ de metástasis en población polaca. Nuestra investigación pudo comprobar en el análisis multivariado que la presencia de enfermedad metastásica constituye un factor pronóstico independiente de menor SLE y SG a los 3 años (15.1\% vs $53.9 \%, p=0.01)$, coincidiendo por lo reportado en otros estudios ${ }^{(4,6,8,15,16)}$, como por ejemplo el del European Intergroup Cooperative Ewing's Sarcoma Study Group (EICESS), donde la sobrevida libre de recaída a los 5 años fue de 22\% para los pacientes con metástasis a diferencia del $55 \%$ de los sin metástasis al diagnóstico ${ }^{(5)}$.

Aunque ya se ha demostrado de que la presencia de metástasis es un factor pronóstico de menor SG, los resultados del presente estudio muestran que lamentablemente nuestros pacientes tienen una menor SG comparada con otras poblaciones (Tabla 5), en donde la SG para pacientes con enfermedad localizada oscila entre $57-81 \%$ y en pacientes con metástasis entre 25$42 \%$, mientras que nuestra investigación determinó que la SG para enfermedad localizada fue de $53.9 \%$ y para enfermedad metastásica del $15.1 \%$, valores muy inferiores a los alcanzados a nivel internacional, tal vez esto se relacione con la mayor cantidad de pacientes que presentan metástasis al debut de la enfermedad.

Tabla 5. Sobrevida Global del Sarcoma de Ewing en enfermedad

\begin{tabular}{rrr}
\multicolumn{1}{c}{ Sobrevida Global (SG) } & \multicolumn{1}{c}{$\begin{array}{c}\text { Enfermedad } \\
\text { Localizada }\end{array}$} & $\begin{array}{r}\text { Enfermedad } \\
\text { Metastásica }\end{array}$ \\
\hline Rodríguez-Galindo et al (a los 5 años) & $38.2 \%$ \\
Karski et al (a los 5 años) & $74.2 \%$ & $25-40 \%$ \\
Raciborska et al (a los 5 años) ${ }^{(12)}$ & $57-81 \%$ & $42 \%$ \\
Duchman et al (a los 10 años) & $68 \%$ & $28.1 \%$ \\
Rios et al (a los 3 años) & $66.8 \%$ & $15.1 \%$
\end{tabular}

El tamaño tumoral por otra parte, constituye un factor de mal pronóstico tanto para la SG y SLE, el $72 \%$ de nuestros pacientes tuvo una lesión tumoral $\geq 5 \mathrm{~cm}$, similar a lo publicado por Huh et $\mathrm{al}^{(16)}$, donde el $74 \%$ de su población tenía una lesión tumoral $\geq 5 \mathrm{~cm}$. Sin embargo no hay un estándar en cuanto al punto de corte, lo que si se demostró es que entre más grande la lesión tumoral peor SLE y SG, por ejemplo en el estudio de Duchman et al ${ }^{(15)}$ la sobrevida a los 10 años fue de $66.8 \%$ para tumores $<5 \mathrm{~cm}$ y de $41.7 \%$ para tumores $>10 \mathrm{~cm}(\mathrm{p}=<0.001)$, por su parte, RodríguezGalindo et al $^{(4)}$ reportó que una lesión tumoral $\geq 8 \mathrm{~cm}$ tenía una menor SG a los 5 años (50.7\% Vs 57.6, p=0.010), a su vez, Oberlin et $a^{(12)}$ de la Sociedad Francesa de Oncología Pediátrica, indicó que un tumor $\geq 8 \mathrm{~cm}$ presentaba menor SLE a los 5 años ( $p=0.002)$, en lo que se refiere a nuestro estudio, se demostró que la SLE y SG a los 3 años de seguimiento eran menores si la lesión tumoral medía $\geq 5 \mathrm{~cm}$ $(p=0.01)$.

$\mathrm{Si}$ bien algunos estudios mencionan que la ubicación extraósea de los TFSE tiene mejor sobrevida global que los TFSE óseos ${ }^{(17)}$, esto no se replicó en nuestro estudio, en donde la SLE y la SG no tuvieron diferencias estadísticamente significativas ni el análisis univariado ni el multivariado. Asimismo pudimos observar que en nuestro estudio la ubicación pélvica del tumor no se relacionó con una menor
SLE o SG, a diferencia de lo publicado por Gupta et al ${ }^{(18)}$ quienes reportaron una SLE y SG a los 3 años menor para aquellos pacientes con enfermedad pélvica $(p=0.018)$ y de lo publicado por el Intergroup Ewing's Sarcoma Study (IESS) que reportó que los pacientes con tumores pélvicos tenía menores índices de sobrevida comparado con aquellos que tenían lesiones en extremidades ${ }^{(19)}$ (Tabla 2 y 3 ).

En cuanto al tratamiento local del tumor, se realizó cirugía en el $75 \%$ de los pacientes, el $27 \%$ tuvo márgenes comprometidos, sin embargo esto no se asoció con peor SLE o SG, similar a lo reportado en otras publicaciones ${ }^{(16)}$. La radioterapia combinada con la cirugía fue la forma de tratamiento local más frecuente en nuestro estudio (65.5\%), siendo igual la cantidad de pacientes que recibieron sólo cirugía o sólo radioterapia (10.3\%), diferente a lo publicado por Raciborska et al $^{(13)}$ en cuyo estudio el $53 \%$ recibió sólo cirugía mientras que el $25.7 \%$ recibió la terapia combinada, por su parte Rodríguez-Galindo et $\mathrm{al}^{(4)}$, reportaron que el $25 \%$ de los pacientes recibió terapia combinada, el 19\% sólo cirugía y el $55 \%$ sólo radioterapia.

Finalmente con respecto al estadio clínico como factor pronóstico, este no fue estadísticamente significativo en nuestro estudio, lo que podemos resaltar que nuestra sobrevida global de 0.13 para el estadio clínico IV (Figura 5), 
está por debajo de lo reportado por Paulussen et al ${ }^{(20)}$ en el European Intergroup Cooperative Ewing Sarcoma Studies, quienes tuvieron una sobrevida de 0.27 para los pacientes con estadio clínico IV.

Dentro las limitaciones de la investigación, tenemos que se trató de un estudio retrospectivo, y con número reducido de casos a pesar de lo largo tiempo de estudio comprendido (10 años), y que sólo evaluó a pacientes niños y adolescentes hasta los 18 años, no incluyéndose en el estudio a los adultos jóvenes, puesto que la Unidad de Oncología Pediátrica y del Adolescente sólo atiende a pacientes hasta los 18 años de edad; por otro lado tiene la fortaleza de ser el primer reporte de TFSE a nivel nacional, y que además no tuvo pérdida de casos en el seguimiento.

En conclusión, los TFSE son neoplasias de mal pronóstico, que se desarrollan mayormente en los niños y adolescentes, nuestro estudio determinó que los factores que contribuyen a una menor SLE y SG son la presencia de metástasis al debut de la enfermedad y un tamaño tumoral $\geq 5 \mathrm{~cm}$, lamentablemente la mayoría de nuestros pacientes tuvieron ambas características lo que resulto en muy baja SLE y SG. Es necesario realizar un diagnóstico precoz que permita que los pacientes reciban tratamiento oportuno ya que TFSE que son localizados y de menor tamaño tienen mejor pronóstico.

\section{REFERENCIAS BIBLIOGRÁFICAS}

1. Balamuth N, Womer R. Ewing's sarcoma. Lancet Oncol. 2010;11(2):184-92.

2. Madero L. Sarcoma de Ewing. En: Madero L, Lassaletta A, Sevilla J, editores. Hematología y oncología pediátrica. 3a Edición. Madrid: Ergon; 2015. p. 651-63.

3. MINSA - Instituto Nacional de Enfermedades Neoplásicas. Registro de cáncer de Lima Metropolitana, Incidencia y Mortalidad 2010-2012.pdf [Internet]. [citado el 25 de setiembre del 2017]; Disponible en: http://bvs.minsa.gob.pe/local/ MINSA/3774.pdf

4. Rodríguez-Galindo C, Liu T, Krasin M, Wu J, Billups C, Daw N, et al. Analysis of prognostic factors in ewing sarcoma family of tumors. Cáncer. 2007;110(2):375-84.

5. Cotterill $S$, Ahrens $S$, Paulussen $M$, Jürgens $H$, Voûte $P$, Gadner $\mathrm{H}$, et al. Prognostic Factors in Ewing's Tumor of Bone: Analysis of 975 Patients From the European Intergroup Cooperative. Journal of Clinical Oncology. 2000; 18(17): 3108-14.

6. Verma V, Denniston KA, Lin CJ, Lin C. A Comparison of Pediatric vs. Adult Patients with the Ewing Sarcoma Family of Tumors. Front Oncol. 2017;7: 82

7. Karski EE, Mcllvaine E, Segal MR, Krailo M, Grier HE, Granowetter $L$, et al. Identification of Discrete Prognostic Groups in Ewing Sarcoma. Pediatr Blood Cancer. 2016;63(1):47-53.

8. Bellan D, Garcia R, Garcia J, Petrilli M, Viola D, Schoedl M, et al. et al. Sarcoma de ewing: epidemiología e prognóstico dos pacientes tratados no instituto de oncología pediátrica, iop-graacc-unifesp. Rev Bras Ortop [Internet] 2012 [citado el 27 de julio del 2017]; Disponible en: http://www.repositorio. unifesp.br/handle/11600/6813

9. Xie C-F, Liu M-Z, Xi M. Extraskeletal Ewing's sarcoma: a report of 18 cases and literature review. Chin J Cáncer 2010;29(4):420-4.

10. Delaplace M, Lhommet C, de Pinieux G, Vergier B, de MuretA, Machet L. Primary cutaneous Ewing sarcoma: a systematic review focused on treatment and outcome. Br J Dermatol. 2012;166(4):721-6.
11. Sadiq M, Ahmad I, Shuja J, Ahmad K. Primary Ewing sarcoma of the kidney: a case report and treatment review. CEN Case Rep.2017:1-4

12. Oberlin O, Deley M, Bui B, Gentet J, Philip T, Terrier P, et al. Prognostic factors in localized Ewing's tumours and peripheral neuroectodermal tumours: the third study of the French Society of Paediatric Oncology (EW88 study). Br J Cancer. 2001; 85(11): 1646-54.

13. Raciborska A, Bilska K, Drabko K, Chaber R, Sobol G, Pogorzała $M$, et al. Validation of a multi-modal treatment protocol for Ewing sarcoma-A report from the polish pediatric oncology group. Pediatr Blood Cancer. 2014; 61(12): 2170-4.

14. Bacci G, Forni C, Longhi A, Ferrari S, Donati D, De Paolis M, et al. Long-term outcome for patients with non-metastatic Ewing's sarcoma treated with adjuvant and neoadjuvant chemotherapies. 402 patients treated at Rizzoli between 1972 and 1992. Eur J Cancer Oxf Engl. 2004;40(1):73-83.

15. Duchman K, Gao Y, Miller B. Prognostic factors for survival in patients with Ewing's sarcoma using the surveillance, epidemiology, and end results (SEER) program database. Cancer Epidemiol. 2015;39(2):189-95.

16. Huh W, Daw N, Herzog C, Munsell M, McAleer M, Lewis V. Ewing sarcoma family of tumors in children younger than 10 years of age. Pediatr Blood Cancer. [Internet] 2017 [citado el 27 de julio del 2017]; 64(4). Disponible en: http://onlinelibrary. wiley.com/doi/10.1002/pbc.26275/abstract

17. Applebaum M, Worch J, Matthay K, Goldsby R, Neuhaus J, West $D$, et al. Clinical features and outcomes in patients with extraskeletal ewing sarcoma. Cancer. 2011;117(13):3027-32.

18. Gupta A, Pappo A, Saunders N, Hopyan S, Ferguson P, Wunder $J$, et al. Clinical outcome of children and adults with localized Ewing sarcoma. Cancer 2010;116(13):3189-94.

19. Kissane J, Askin F, Foulkes M, Stratton L, Shirley S. Ewing's sarcoma of bone: clinicopathologic aspects of 303 cases from the Intergroup Ewing's Sarcoma Study. Hum Pathol. 1983;14(9):773-9.

20. Paulussen $M$, Ahrens S, Burdach S, Craft A, DockhornDworniczak B, Dunst J, et al. Primary metastatic (stage IV) Ewing tumor: survival analysis of 171 patients from the EICESS studies. European Intergroup Cooperative Ewing Sarcoma Studies. Ann Oncol Off J Eur Soc Med Oncol. 1998;9(3):275-81.

Fuentes de financiamiento:

Este artículo ha sido financiado por los autores.

Conflictos de interés:

Los autores declaran no tener ningún conflicto de interés.

\section{Correspondencia:}

Ligia Estefanía Ríos López

Dirección: Jr. Pichincha 667 - Breña

Teléfono: 982077225

Correo electrónico: literly@hotmail.com

Recibido: 25 de agosto de 2017 Evaluado: 27 de agosto de 2017 Aprobado: 17 de octubre de 2017

(c) La revista. Publicado por Universidad de San Martín de Porres, Perú. (cc) Br Licencia de Creative Commons Artículo en acceso abierto bajo términos de Licencia Creative Commons Atribución 4.0 Internacional. (http://creativecommons.org/licenses/by/4.0/) 